\title{
Pain in adolescents and its risk factors: A case-control study
}

\author{
Gonzalo Agüero, M.D. ${ }^{a}$, Soledad Salmain, M.D. ${ }^{b}$, Belén Manzur, M.D. ${ }^{b}$ and \\ Enrique Berner ${ }^{a}$
}

\begin{abstract}
Introduction. The most common painful syndromes (headache, abdominal pain and musculoskeletal pain) develop or worsen during adolescence and are a common reason for consultation.

Objective. Evaluate the association of age, sex, obesity, pubertal development, schooling level, employment and family structure with consultation for pain in adolescents.

Population and methods. Case-control study conducted between February 1st, 2014 and June 30th, 2015.

Inclusion criteria: ages 10 to 20 years, both sexes, consultation for pain (cases), or a checkup and / or school physical (controls). $\chi^{2}$ test and Student's tests were used. Odds ratios (OR) were calculated. A binary logistic regression model was constructed to independently assess each pain-related variable.

Results. A total of 4224 medical records were evaluated; 237 cases and 468 controls were included. Adolescents with pain exhibited: greater age $(\mathrm{p}<0.0001$; OR 2.3 ; 95\% CI: 1.6$3.2)$; greater number of females $(\mathrm{p}<0.0001$; OR 2.24; 95\% CI: 1.61-3.12); greater pubertal development ( $<<0.0035$; OR 2.16; 95\% CI: 1.33.6); greater school dropout level ( $\mathrm{p}<0.0001$; OR 13.4; 95\% CI: 3.9-42.9); greater employment levels ( $\mathrm{p}<0.0001$; OR 3.04; 95\% CI: 1.7-5.3). Only age, female sex and school dropout were independently associated with consultation for pain. There were no significant differences with obesity and family structure.

Conclusion. Older age, female sex and school dropout were independent risk factors in consultation for pain in adolescents. Puberty and employment were associated, but were not found to be independent risk factors.

Key words: chronic pain, headache, musculoskeletal pain, abdominal pain, adolescent.
\end{abstract}

http: / / dx.doi.org/10.5546/ aap.2018.eng.112

To cite: Agüero G, Salmain S, Manzur B, et al. Pain in adolescents and its risk factors: A case-control study. Arch Argent Pediatr 2018;116(2):112-118.

\section{INTRODUCTION}

The International Association for the Study of Pain defines pain as an unpleasant sensory and emotional experience associated with actual or potential tissue damage. ${ }^{1}$ Pain is a common reason for consultation in adolescence. ${ }^{2}$ The most common painful syndromes, such as headache, abdominal pain and musculoskeletal pain (MSP), begin or worsen during this stage. The increase in its prevalence correlates directly with the pubertal development phase ${ }^{3}$ and with age. ${ }^{4}$ Obesity is another factor associated with the presence of pain, particularly with chronic pain in children and adolescents. ${ }^{5}$ In addition, it is more common in females. ${ }^{4,6}$

Swain et al. evaluated adolescents aged 11 to 15 years from the "Health Behaviour in School-aged Children: WHO Collaborative Cross-National Survey (HBSC)." They showed that pain was common in this population: headache in 54\% $(95 \%$ confidence interval [95\% CI]: 54.0$54.3)$; abdominal pain in $49.8 \%$ (95\% CI: 49.6-49.9) and low back pain in 37\% (95\% CI: 36.8-37.1). The three pains were more prevalent in girls and older adolescents. Multiple pains were more common in girls and their prevalence increased directly with age. ${ }^{7}$ This reinforces the hypothesis that pain in adolescence is a major predictor of pain in adult life, which is associated with high health costs and alterations in the quality of life. ${ }^{8}$

Primary headaches (migraine and tension-type headache) are the most common ones. Both prevail in girls since puberty and tend to exhibit a recurrent pattern. ${ }^{9,10}$ Chronic abdominal pain is mainly caused by functional disorders. Their incidence decreases with age in males but not in females. They are usually associated 
with psychosomatic manifestations. ${ }^{11,12}$ For its part, MSP prevalence correlates directly with age. Its most common causes in adolescence are trauma, overuse syndrome and low back pain. ${ }^{13-15}$

Pain is a frequent reason for consultation in adolescents or their families, particularly when its pattern of occurrence is chronic or recurrent. In Argentina, data in this respect are scarce. In international bibliography there is controversy regarding the influence of psychosocial factors on pain. ${ }^{4}$ In our Department of Adolescence, in 1995, pain was found to be an important reason for consultation and the most commonly reported complaints were headache, chest pain and recurrent abdominal pain. ${ }^{16}$

In this study, we performed a retrospective analysis of the most common painful syndromes as reason for consultation during adolescence (headache, MSP, abdominal pain), and described some related risk factors.

\section{OBJECTIVES}

- Describe age, sex, body mass index (BMI) and pubertal development of adolescents who consulted for pain.

- Assess the degree of association with potential risk factors: age, female sex, obesity, advanced pubertal development, schooling, employment and family structure.

\section{POPULATION, MATERIALS AND METHODS}

The study was conducted at the Department of Adolescence of the Hospital de Agudos Dr. Cosme Argerich, of the Autonomous City of Buenos Aires, between February 1 1', 2014 and June 30 $0^{\text {th }}, 2015$.

Design: Prevalent case-control study. Data were retrospectively obtained.

Inclusion criteria: 1) Cases. Adolescents aged 10 to 20 years, of both sexes, who presented with painful syndrome (headache, abdominal pain, MSP, or their combinations) between January $1^{\text {st }}, 2008$ and December 31 $1^{\text {st }}, 2013.2$ ) Controls. Adolescents who presented at the institution for a check-up and/or a school physical in the same period, and reported no pain at consultation.

Exclusion criteria: Acute febrile illness, trauma, rheumatic and oncohematologic diseases, precocious and delayed puberty, pregnancy, intellectual disability or acute psychiatric disease, missing data in medical record (age, sex, weight, height and / or pubertal development stage).

Patients who presented for a health check-up or a school physical and, in addition, reported experiencing pain, were included as cases.

\section{Description of variables}

Dependent variables (pain)

Type, quantity and duration were recorded. Consultations for headache, abdominal pain, MSP, or multiple pain syndrome were evaluated. "Multiple pains" were defined as the combination of, at least, two painful syndromes mentioned above or two anatomically unrelated MSPs. Depending on the duration of the symptom, development was divided into acute $(<12$-week course) and chronic ( $\geq 12$-week duration).

\section{Independent variables (exposure)}

1) Age: In years-old actually reached. 2) Sex: Male and female. 3) Anthropometric data and pubertal development: In our Department of Adolescence, these were recorded in accordance with the recommendations of the Argentine Society of Pediatrics. ${ }^{17}$ Based on the BMI $(\mathrm{kg} /$ $\mathrm{m}^{2}$ ) percentile, patients were classified as with and without obesity. Pubertal development was assessed according to the Tanner stages, and was classified as prepubertal stage (breast development and genital development - B / G - I: Pubertal development has not begun) and pubertal stages (B/G II, III, IV and V: Pubertal development has begun). If pubertal development was not assessed at the first visit, data from the second visit was collected, as long as it took place within 30 days. 4) Schooling: Schooling, school overage (over the traditional school age for their level due to retention and / or dropout) and school dropout (abandoning school without having completed the primary and / or secondary cycles). 5) Employment: Any remunerative or non-remunerative activity performed by an adolescent implying the existence of an employment relationship. 6) Family structure: Based on the genogram, families were classified into single and two-parent families with one or more than one child.

Control of selection biases: Prevalent cases were recorded, regardless of their severity or time of development; medical records were classified according to the inclusion and exclusion criteria described above.

\section{Statistical analysis}

Sample: The sample size needed to contrast the null hypothesis (so that the odds ration -ORwas equal to 1) was calculated. Of the variables to be assessed, obesity was the less prevalent variable and was taken as the basis for sample size determination. Six percent of the adolescent population was estimated to be obese ${ }^{18}$ and an 
OR of 3, with a confidence of $95 \%$, a power of $90 \%$ and a case/ control ratio of 1:2, was deemed to be a significant difference. With these data, the Epidat version 4.1 software defined a sample size for the $\chi^{2}$ test, with Yates correction for continuity, of 161 cases and 322 controls.

Data collection: Data were retrospectively obtained from the admission medical records, randomly chosen with a sampling fraction of $1 / 3$.

Statistical analysis: For qualitative variables, frequencies, percentages and 95\% CI were calculated. The independence $\left(\chi^{2}\right)$ test was used to compare them. For quantitative variables, the central distribution variables (mean, median and mode), standard deviation, minimum, maximum and range were calculated. The Student's test was used to compare them when data distribution was normal. When variables did not show normal distribution or variance homogeneity and / or were highly asymmetrical, non-parametric statistical tests were used. ORs were calculated to evaluate association with risk factors. Since some variables might mutually influence one another, and to avoid confusion biases, a binary logistic regression model was constructed to assess the independent weight of the association of each risk factor with the presence of pain. In the logistic regression we included the variables that were significantly associated $(p<0.05)$ with consultation for pain in the bivariate analysis.

Data processing: Epidat version 4.1 software (Government of Galicia, Spain, Pan American Health Organization, 2012).

Ethical aspects: Approval was obtained from the hospital Ethics Committee. No informed consent was requested because data were obtained from medical records, without the patients' direct participation. Patients were identified with sequential numbers, and their personal details were not recorded to safeguard their privacy.

\section{RESULTS}

A total of 4224 medical records were examined and the calculated sample size was attained. The survey included 237 cases and 492 controls (Figure 1). Painful syndromes found: headache in 27\% (95\% CI: 20.7-32.4); abdominal pain in 25\% (95\% CI: 19.2-30.6); MSP in 30\% (95\% CI: 24.336.4) and multiple pains in 18\% (95\% CI: 13-23.3). The chief complaint in $47.7 \%$ (95\% CI: 41.154.2) of consultations was chronic pain. Chronic development was more common in the multiple pain syndrome $(67 \%)$, followed by headache $(50.8 \%)$, abdominal pain $(48.1 \%)$ and MSP (38\%).

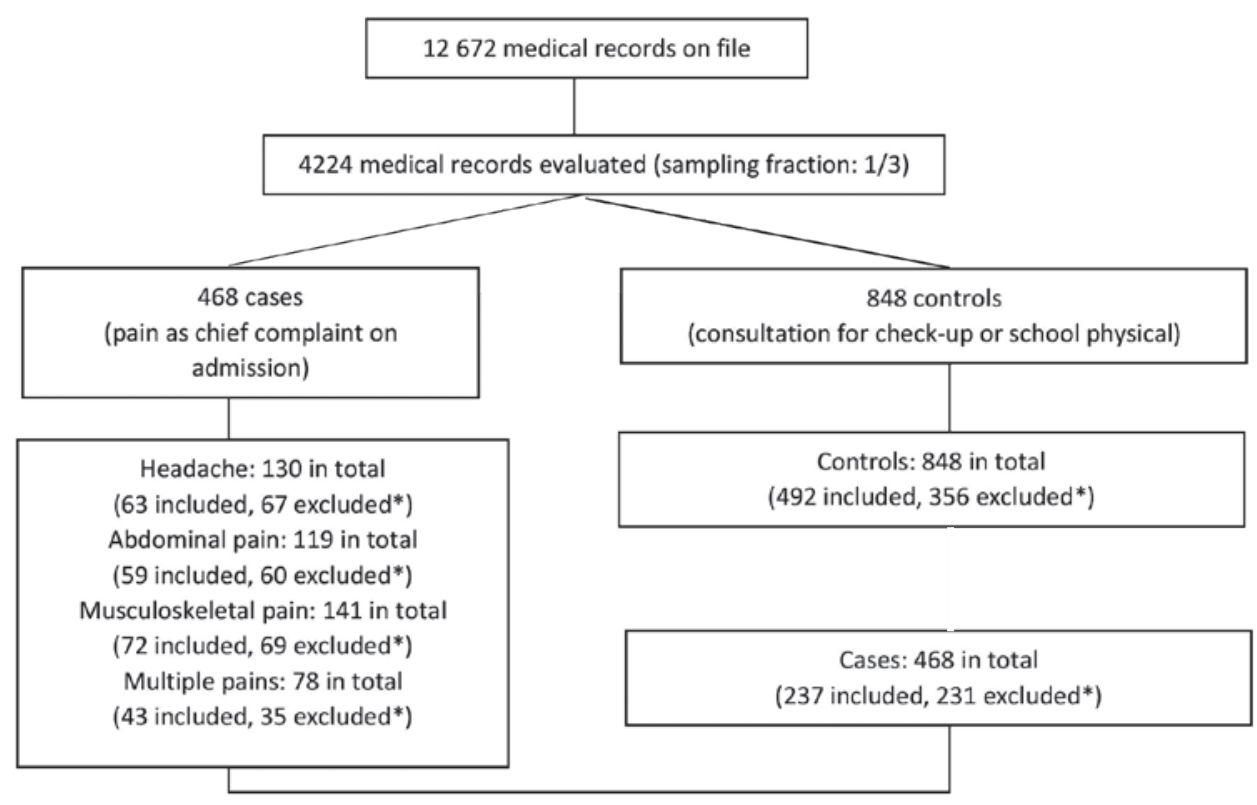

${ }^{*}$ Controls excluded: 307 due to missing data about pubertal development, 19 due to fever, 13 due to trauma, 15 due to lack of other data (weight, height), 2 due to rheumatic disease.

${ }^{* *}$ Cases excluded: 204 due to missing of data about pubertal development, 12 due to fever, 8 due to trauma, 7 due to lack of other data (weight, height, age). 
In multiple pain syndromes, mixed development (acute/chronic) was found in 7\%. Differences in time of development were statistically significant $(\mathrm{p}=0.0039)$.

The exposure variables evaluated are shown in Table 1.

Age (Figure 2). The mean age was greater in the group with pain: $14.2 \pm 2.9$ (10-20 range) years versus $13.2 \pm 2.5$ (10-20 range) years, $\mathrm{p}=0.0055$. After dividing both groups into $<15$ years and $\geq 15$ years, we also noted that cases had significantly greater age than controls $(\mathrm{p}<0.0001$; OR 2.3; 95\% CI: 1.6-3.2).

Sex. There were more women in cases $(70 \%$; 95\% CI: 63.9-76.1) than in controls $(51 \% ; 95 \%$ CI: 46.7-55.7); the difference was statistically significant ( $p<0.0001$; OR 2.24; 95\% CI: 1.61-3.12).

Pubertal development. In cases, there were more adolescents whose pubertal development had begun $(92 \% ; 95 \%$ CI: 88.3-95.6) than in controls (84\%; 95\% CI: 80.5-87.3). This difference was statistically significant $(\mathrm{p}=0.0035$; OR 2.16; 95\% CI: 1.27-3.66).

Obesity (Figure 3). The BMI showed no significant differences between cases $(22.1 \pm 4)$ and controls $(21.2 \pm 4) ; p=0.97$. Neither did the obesity percentage show significant differences between both groups: $15.6 \%$ in cases and $18.1 \%$ in controls ( $p=0.47$; OR 0.84; 95\% CI: 0.55-1.27).

Education. Cases exhibited greater school overage: $35.4 \%$ (95\% CI: 29.1-41.7) versus $24.6 \%$ (95\% CI: 20.7-28.5): $\mathrm{p}=0.0023$; OR $1.68(95 \%$ CI: 1.20-2.35). Likewise, they evidenced a greater dropout percentage: $7.6 \%$ (95\% CI: 4-11.2) versus $0.6 \%$ (95\% CI: $0.1-1.7$ ): $\mathrm{p}<0.0001$; OR 13.4 (95\% CI: 3.9-42.9). Differences were statistically significant.

Employment. There were more working adolescents in controls, $13.5 \%$ (95\% CI: 8.9-18) versus $4.9 \%$ (95\% CI: 2.8-6.8): $\mathrm{p}=0.0001$; OR 3.04 (95\% CI: 1.75-5.30). After excluding from the analysis the $>18$ year-olds who had not dropped out from school, the difference between groups remained significant $(\mathrm{p}<0.0001$; OR $3.5 ; 95 \%$ CI: 1.9-6.3).

Family structure. There were no significant differences in parental structure (two-parent, single-parent): $\mathrm{p}=0.362$; OR 0.84 (95\% CI: 0.591.18). Neither were differences found between those who lived with and without siblings ( $\mathrm{p}=0.842$; OR 0.94, 95\% CI: 0.61-1.42).

Binary logistic regression (Table 2). After the analysis, the variables independently associated with pain were age, sex and school dropout.

TABLE 1. Bivariate analysis of risk factors

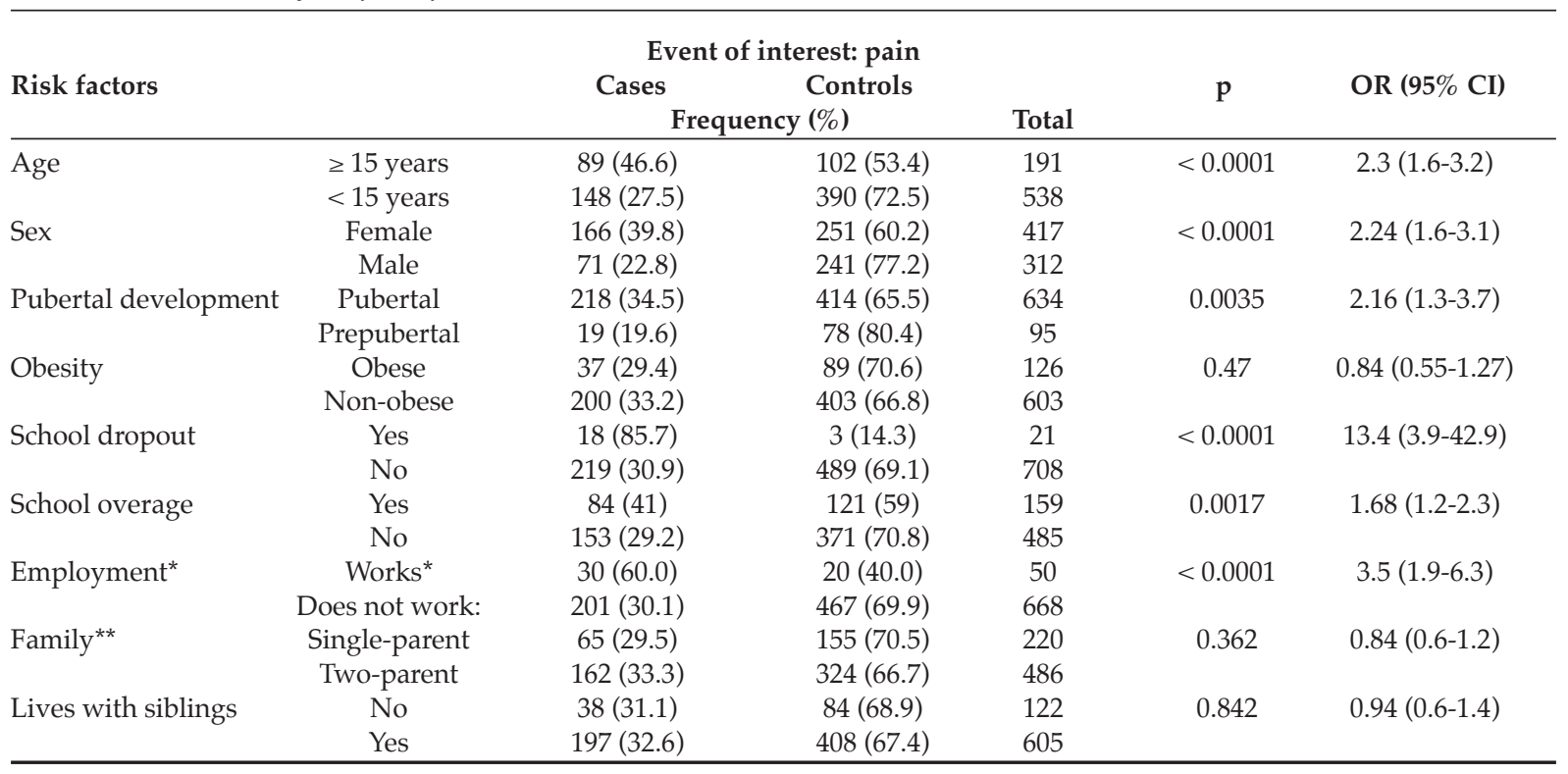

*Employment: 11 unschooled adolescents over the age of 18 years ( 6 cases and 5 controls) were excluded from the analysis as school dropouts were a priori more likely to work.

**Family: 23 adolescents (10 cases and 13 controls) who did not live with their parents for different reasons were excluded from the analysis. 


\section{DISCUSSION}

This study evaluated risk factors associated with common painful syndromes in adolescents. These presented for consultation to a Department of Adolescence belonging to an acute care hospital in the City of Buenos Aires.

Age $\geq 15$ years, female sex and onset of pubertal development doubled the risk of consultation for pain; however, only the first two variables were independent risk factors. Our findings agree with those shown by international literature: women experience pain more commonly than men; prevalence in women increases with age. Debate is under way as to whether this difference is due to biological mechanisms related to puberty or to the contribution of psychosocial factors. ${ }^{4,6,19}$ Logistic regression made it possible to independently assess each variable associated with consultation for pain, as they mutually influence one another. On the one hand, different areas of growth and development, such as age, weight, height and puberty, increase simultaneously and, hence, it is important to assess the influence that each of them has. On the other hand, socioeconomic vulnerability factors tend to coexist, such as the need to work to contribute to the family income and poor academic performance.

Half of the patients assessed experienced chronic pain; the multiple pain syndrome prevailed in this group. Acute development was more common in consultation for isolated
FiguRE 2. Box plot for age (years)

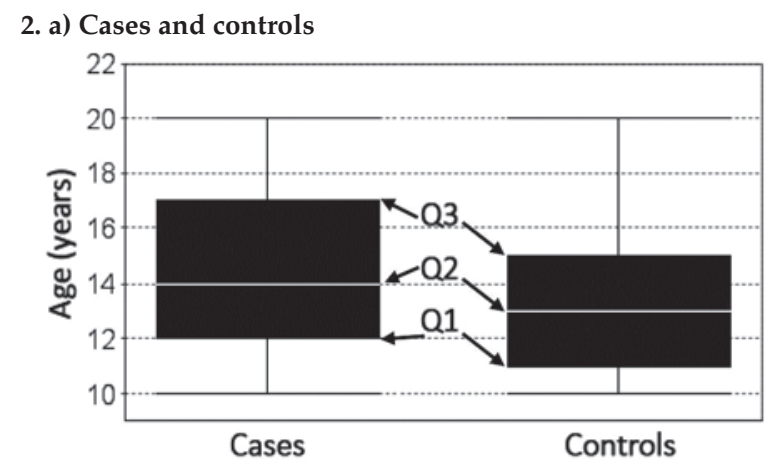

Cases $(n=237)$ and controls $(n=492)$

2. b) Cases discriminated by type of pain

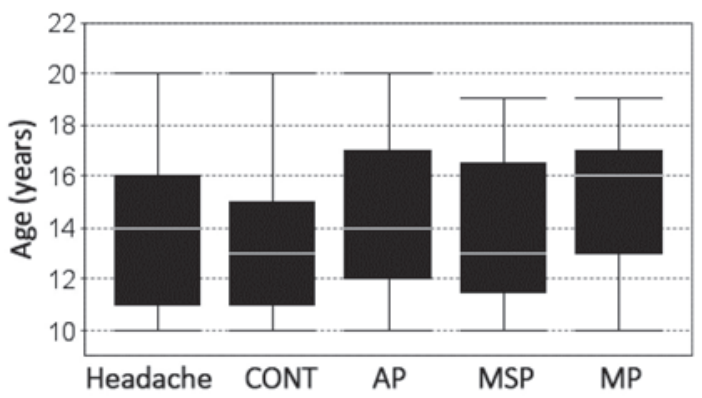

2. b. Cases (discriminated by type of pain) and controls

CONT: controls; AP: abdominal pain; MSP: musculoskeletal pain; MP: multiple pains.

Q1: First quartile (represents 25\% of data); Q2: Second quartile or median (represents $50 \%$ of data); Q3: Third quartile (represents $75 \%$ of data).

2. a: In cases, asymmetric data distribution is observed at the expense of greater age in this group; in controls, distribution is symmetric.

2. $\mathrm{b}$ : The median age for the different types of pain was greater than for controls, except for musculoskeletal pain.
FIgURE 3. Box plot for body mass index

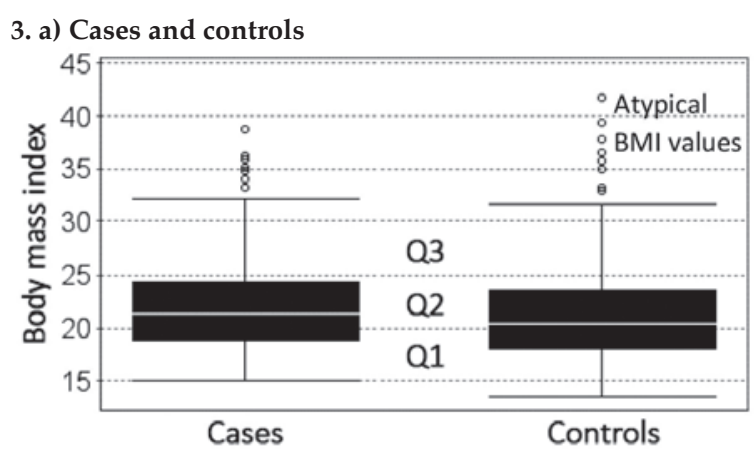

3. a. Total cases $(n=237)$ and controls $(n=492)$

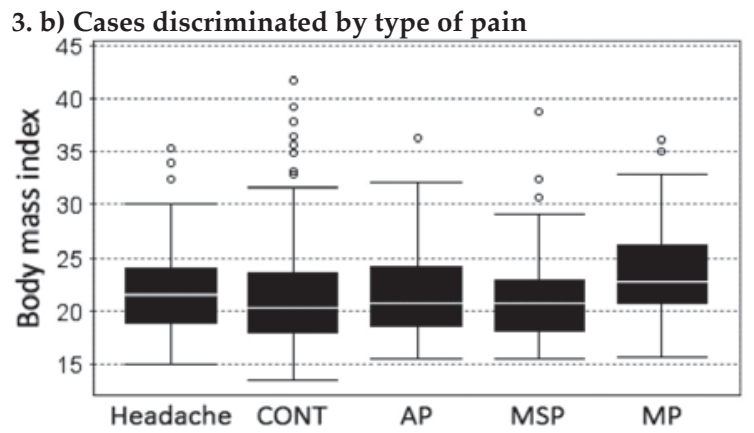

3. b. Cases (discriminated by type of pain) and controls

CONT: controls; AP: abdominal pain; MSP: musculoskeletal pain; MP: multiple pains.

Q1: First quartile (represents 25\% of data); Q2: Second quartile or median (represents $50 \%$ of data); Q3: Third quartile (represents $75 \%$ of data).

Atypical values: correspond to cases of obesity that deviate too much from the main body of data.

3. a: Distribution of BMI in cases and controls was symmetrical, with no significant differences.

3. b: Median BMI for the different types of pains does not differ from controls, except for the case of multiple pains, which showed higher BMI values. 
MSP. Since early childhood, the different painful syndromes have characteristic patterns of change over time. These patterns, called "pain trajectories", show that chronic and recurrent pain is common among adolescents and that different types of pain (headache, abdominal pain and low back pain) tend to coexist. ${ }^{6}$ Similarly, the HUNT survey ${ }^{20}$ shows that most adolescents experiencing chronic pain report pain from more than one body site.

In our study, work increased the risk of consulting for pain, but not independently; however, other authors emphasize the relationship between pain and work. A follow-up of technical school students entering working life showed that multi-site MSP was prevalent (69\%) and stable over time. ${ }^{20}$ Increased mechanical workload and quantitative demands and low socioeconomic status were associated with increased number of MSP sites among women, while tobacco use was found as a risk factor among young men. There is now increased evidence that psychosocial factors, such as high demand levels at work, play an important role in multi-site MSP. ${ }^{21}$

School dropout was associated with a 13-fold higher risk for pain consultation. Persistent or recurrent pain has been described to interfere with academic performance and social activities, and favor the development of anxiety and depression. ${ }^{4,20}$ In any case, pain cannot conclusively be said to be the cause for poor academic performance. As a matter of fact, pain and poor academic performance are probably the product of situations of vulnerability in this group of patients, such as family conflicts, low income, mental health problems, need to work since early ages, among others.

Contrary to what has been described in international literature, ${ }^{5,21}$ no differences were found in the presence of obesity. Since obesity primarily affects individuals of lower educational level and socioeconomic status, ${ }^{22}$ this absence of difference might be due to the origin of our population. Our hospital population includes, mostly, families whose household heads do not have a stable job and/or health coverage.

No differences were observed in the parental structure or co-residence with siblings, although these associations were not clear in other studies either. ${ }^{4}$

Implications of the study for practice and research: chronic pain and multiple pain syndromes are prevalent in adolescence; independent risk factors associated with consultation for pain, such as age, female sex and school dropout, were identified. This findings show that biological, development and socioeconomic factors converge and increase the risks for developing a painful syndrome in adolescence.

Limitations: 1) Limitations that are characteristic of the methodological design (cases and controls), particularly, selection and information bias; yet, its advantages justify its use. 2) The main cause for exclusion was the lack of data about pubertal development in medical records, particularly in older adolescents; this situation might have affected results regarding the influence of puberty. 3) Another possible bias is that controls included more schooled patients; however, it is known that the majority of our patients presenting to our Department of Adolescent Medicine are schooled and that a health check-up is one of the most common reasons for consultation. 4) Our study participants belong to the hospital population and, hence, are not representative of the general population; however, the results obtained are mostly consistent with previous studies.

TABLE 2. Binary logistic regression analysis: assessment of independent risk factors in the consultation for pain

\begin{tabular}{lcccc}
\hline Variable & $\beta$ Coefficient & Odds ratio & $\mathbf{9 5 \%}$ CI & p \\
\hline 15 years old & 0.381 & 1.46 & $1.02-2.1$. & $0.040^{*}$ \\
Female & 0.770 & 2.16 & $1.52-3.07$ & $<0.0001^{*}$ \\
Puberty onset & 0.170 & 1.19 & $0.67-2.11$ & 0.563 \\
School dropout & 2.206 & 9.10 & $2.52-32.69$. & $0.001^{*}$ \\
Works $^{*}$ & 0.592 & 1.81 & $0.96-3.41$. & 0.067 \\
\hline
\end{tabular}

95\% CI: 95\% confidence interval. -2 initial likelihood ratio=919,15; -2 final likelihood ratio= 857.4; likelihood ratio with $\mathrm{p}<0.0001$. Hosmer-Lemeshow test with $\mathrm{p}=0.967$.

*They were independently associated with consultation for pain. 
5) From the cross-sectional observation of data it is not possible to know whether the differences observed persist over time.

Cohort studies of general adolescent population are required to address these shortcomings. It might be interesting to evaluate each type of pain on a separate basis, particularly, in adolescents with multiple pain syndrome, as this group is likely to have distinct clinical characteristics and is not merely the sum of one or more pains.

\section{CONCLUSION}

In adolescents, the risk of presenting to a provider for a painful syndrome has doubled in patients above the age of 15 years and in women. Such risk was 13-fold higher in situations of school dropout. Pubertal development and the need to work also increased the risk, although not independently. Obesity, parental structure and number of siblings did not have a significant influence.

\section{Acknowledgments}

The authors thank Juan Gagliardi, M.D. for his critical reading of the manuscript.

\section{REFERENCES}

1. International Association for Study of Pain. IASP taxonomy. [Accessed on: June 8th, 2016]. Available at: http:/ / www. iasp-pain.org/ Taxonomy?navItemNumber $=576$.

2. Pasqualini D, Llorens A. Síntomas y malestares frecuentes. In:Salud y bienestar de adolescentes y jóvenes: una mirada integral. Buenos Aires: OPS/OMS, 2010.p.455-505.

3. Patton GC, Viner R. Pubertal transitions in health. Lancet 2007;369(9567):1130-9.

4. King S, Chambers C, Huguet A, et al. The epidemiology of chronic pain in children and adolescents revisited. Pain 2011;152(12):2729-38.

5. Wilson A, Samuelson B, Palermo T. Obesity in children and adolescents with chronic pain: Associations with pain and activity limitations. Clin J Pain 2010;26(8):705-11.

6. Dunn K, Jordan K, Mancl L, et al. Trajectories of pain in adolescents: a prospective cohort study. Pain 2011;152(1):66-73.
7. Swain MS, Henschke N, Kamper SJ, et al. An international survey of pain in adolescents. BMC Public Health 2014; $14: 447$.

8. Henschke N, Kamper SJ, Maher CG. The epidemiology and economic consequences of pain. Mayo Clin Proc 2015;90(1):139-47.

9. Hershey A. Current approaches to the diagnosis and management of paediatric migraine. Lancet Neurol 2010;9(2):190-204.

10. Anttila, P. Tension-type headache in childhood and adolescence. Lancet Neurol 2006;5(3):268-74.

11. Banez, G. Chronic abdominal pain in children: what to do following the medical evaluation. Curr Opin in Pediatr 2008;20(5):571-5.

12. Wallis E, Fiks A. Nonespecific abdominal pain in pediatric primary care: Evaluation and outcomes. Acad Pediatr 2015;15(3):333-9.

13. De Inocencio Arocena J. Dolor musculoesquelético en pediatría de atención primaria. Etiología y orientación diagnóstica. Arch Argent Pediatr 2006;104(3):275-83.

14. Balagué F, Mannion A, Pellisé F, et al. Non-specific low back pain. Lancet 2012;379(9814):482-91.

15. Kim H, Green D. Adolescent back pain. Curr Opin Pediatr 2008;20(1):37-45.

16. BianculliC.Asistencia hospitalaria/Criterios innovatorios. In: Servicio de Adolescencia del Departamento Materno Infanto Juvenil, Hospital de Agudos Dr. Cosme Argerich. Proyecto Adolescencia Buenos Aires 1992-93-94, Propuesta para un modelo de atención integral asisitencial-docente intra y extrahospitalario. Buenos Aires: FUSA; 2000; 1995.

17. Comité Nacional de Crecimiento y desarrollo. Guía para la evaluación del crecimiento físico. Sociedad Argentina de Pediatría, 2013. [Accessed on: September 8th, 2017. Availableat:http:/ / www.sap.org.ar/docs/publicaciones/ libro_verde_sap_2013.pdf.

18. Ferrante S, Linetzky B, Ponce M, et al. Prevalencia de sobrepeso, obesidad, actividad física y tabaquismo en adolescentes argentinos: Encuestas Mundiales de Salud Escolar y de Tabaco en Jóvenes, 2007-2012. Arch Argent Pediatr 2014;112(6):496-503.

19. Hoftn GB, Romundstad PR, Zwart JA, et al. Chronic idiopathic pain in adolescence -high prevalence and disability: The Young HUNT study 2008. Pain 2011;152(10):2259-66.

20. Hanvold TN, Lunde LK, Koch M, et al. Multisite musculoskeletal pain among young technical school students entering working life. BMC Musculo skelet Disord 2016;17:82.

21. Smith S, Sumar B, Dixon K. Musculoskeletal pain in overweight and obese children. Int J Obes (Lond) 2014;38(1):11-5.

22. Pedraza DF. Obesidad y pobreza: marco conceptual para su análisis en Latinoamérica. Saúde Soc 2009;18(1):103-17. 